Please do not remove this page

RMIT

UNIVERSITY

\title{
Enhanced single-photon emission in the near infrared from a diamond color center
}

Aharonovich, I; Zhou, Chunyuan; Stacey, Alastair; Orwa, J.; Castelletto, Stefania; Simpson, D; Greentree, Andrew

https://researchrepository.rmit.edu.au/esploro/outputs/9921858436501341/filesAndLinks?institution=61RMIT_INST\&index=null

Aharonovich, I., Zhou, C., Stacey, A., Orwa, J., Castelletto, S., Simpson, D., Greentree, A., Treussart, F., Roch, J. ., \& Prawer, S. (2009). Enhanced single-photon emission in the near infrared from a diamond color center. Physical Review B (Condensed Matter and Materials Physics), 79(23), 1-5.

https://doi.org/10.1103/PhysRevB.79.235316

Document Version: Published Version

Published Version: https://doi.org/10.1103/PhysRevB.79.235316

Repository homepage: https://researchrepository.rmit.edu.au

(c) 2009 The American Physical Society

Downloaded On 2023/04/26 21:35:51 +1000 
Thank you for downloading this document from the RMIT Research Repository.

The RMIT Research Repository is an open access database showcasing the research outputs of RMIT University researchers.

RMIT Research Repository: http://researchbank.rmit.edu.au/

\section{Citation:}

Aharonovich, I, Zhou, C, Stacey, A, Orwa, J, Castelletto, S, Simpson, D, Greentree, A, Treussart, F, Roch, J and Prawer, S 2009, 'Enhanced single-photon emission in the near infrared from a diamond color center', Physical Review B (Condensed Matter and Materials Physics), vol. 79, no. 23, 235316, pp. 1-5.

See this record in the RMIT Research Repository at:

http://researchbank.rmit.edu.au/view/rmit:15484

Version: Published Version

Copyright Statement: (C) 2009 The American Physical Society

Link to Published Version:

http://dx.doi.org/10.1103/PhysRevB.79.235316 


\title{
Enhanced single-photon emission in the near infrared from a diamond color center
}

\author{
Igor Aharonovich, ${ }^{1, *}$ Chunyuan Zhou, ${ }^{2}$ Alastair Stacey, ${ }^{1}$ Julius Orwa, ${ }^{1}$ Stefania Castelletto, ${ }^{1}$ David Simpson, ${ }^{3}$ \\ Andrew D. Greentree, ${ }^{1}$ François Treussart, ${ }^{2}$ Jean-Francois Roch, ${ }^{2}$ and Steven Prawer ${ }^{1,3}$ \\ ${ }^{1}$ School of Physics, University of Melbourne, Melbourne, Victoria 3010, Australia \\ ${ }^{2}$ Laboratoire de Photonique Quantique et Moléculaire, CNRS UMR 8537, Ecole Normale Supérieure de Cachan, \\ 94235 Cachan Cedex, France \\ ${ }^{3}$ Quantum Communication Victoria, Melbourne, Victoria 3010, Australia
}

(Received 15 December 2008; revised manuscript received 12 March 2009; published 15 June 2009)

\begin{abstract}
Individual color centers in diamond are promising for near-term quantum technologies including quantum key distribution and metrology. Here we show fabrication of an as-yet uncharacterized nickel-related complex in diamond which has photophysical properties surpassing the two main-stay centers for single-photon applications, namely, the nitrogen-vacancy and the nickel-nitrogen complex (NE8) center. This center was fabricated using focused ion-beam implantation of nickel into isolated chemical vapor-deposited diamond crystals. A possible correlation of the center to a Ni/Si complex is substantiated by a coimplantation of $\mathrm{Ni}$ and $\mathrm{Si}$ into a pure bulk diamond. Room-temperature photoluminescence studies reveal a narrow emission in the near infrared region centered at $768 \mathrm{~nm}$ with a lifetime as short as $2 \mathrm{~ns}$.
\end{abstract}

DOI: 10.1103/PhysRevB.79.235316 PACS number(s): 61.66.-f, 42.50.Dv, 78.55.-m, 81.05.Uw

\section{INTRODUCTION}

The discovery in 1997 that diamond color centers could be imaged and investigated at individual level ${ }^{1}$ has been highly disruptive to the field of quantum optics. The most well-studied and utilized defect to date has been the negatively charged nitrogen-vacancy (NV) color center, which has been used for demonstrations of quantum key distribution, ${ }^{2}$ single-photon interference, ${ }^{3}$ and is the source in the first commercialized single-photon source. ${ }^{4}$ In addition, the potential of NV centers has been identified in a range of applications including quantum computers and quantum simulators (see, for example, the recent review ${ }^{5}$ ), highprecision magnetometry with their unique spin properties, ${ }^{6}$ and as flexible sources for entangled photons. ${ }^{7}$

Color centers in diamond are highly suitable for such quantum applications because of their combination of desirable properties including photostability, high Debye temperature, and room temperature operation. At present, there are three leading single-photon sources in diamond. These are the NV center, the nickel-related NE8 center, ${ }^{8}$ and the silicon vacancy $\mathrm{Si}-\mathrm{V}$ center. ${ }^{9}$ The latter two exhibit very short lifetimes of 2-3 ns; however, $\mathrm{Si}-\mathrm{V}$ has a long-lived shelving state which reduces its fluorescence intensity. ${ }^{9}$ The narrow emission of the NE8 center around $800 \mathrm{~nm}$ makes is promising for quantum key distribution (QKD) applications.

Recently, ion implantation techniques were used to fabricate color centers in diamond. ${ }^{10-12}$ Using ion implantation of nickel, we address some of the challenges toward spatially controlled fabrication of nickel-related single-photon centers in diamond. Furthermore, combining a dual scanning electron microscope and a focused ion beam (SEM/FIB) to create single-photon emitters in a desired crystal and in a preferred position constitutes significant progress toward a scalable control and fabrication of multiqubit quantum optic devices (e.g., $Q$-switched quantum gates ${ }^{13}$ ) by affording the ability to locate implantation zones within optical nanostructures. It may even give rise to a "step and repeat" technology, ${ }^{14}$ which would be of great benefit to large-scale integrated quantum devices.

Given the potential of nickel-related defect centers, there have been many attempts at their controlled fabrication in diamond. Previously demonstrated techniques of incorporation of nickel into chemical vapor deposition (CVD) diamond films by seeding the substrate with a diamond/nickel powder ${ }^{15}$ or implanting the nickel into the substrate onto which the diamond crystals were subsequently grown ${ }^{16}$ did not allow a controlled formation of NE8 centers in a specific diamond crystal and in a preferred location. Furthermore, the interaction between various impurity atoms within the diamond lattice (such as nitrogen and nickel or silicon and nickel) is still unclear which makes the controlled fabrication of optical centers a challenging task. Indeed there are no reports demonstrating the formation of NE8 centers due to nickel implantation.

In this work we demonstrate the formation of nickelrelated single-photon emitters in a controllable manner by a FIB implantation of nickel into individual CVD diamond nanocrystals. The use of a dual SEM/FIB allows for the imaging of a specific crystal prior to implantation with a precise accuracy suitable for past processing and scalability. This method allows fabrication of optical centers in a specific crystal of choice as well as in a given space, in contrast to previous approaches where the incorporation of the nickel into diamond crystal was not dependent on the crystal selection. ${ }^{15,16}$ The formed centers are not NE8 but show surprising and potentially important properties including an estimated near unity quantum efficiency, a near infrared emission $(768 \mathrm{~nm})$, and a $2 \mathrm{~ns}$ radiative lifetime. A further coimplantation of $\mathrm{Ni}$ and $\mathrm{Si}$ atoms into pure bulk diamond indicates that the defect center belongs to an unreported class of nickel-silicon composite centers. The simple fabrication method and the extended optical properties make this defect a very attractive candidate for integration with external microstructures. The coimplantation of $\mathrm{Ni} / \mathrm{Si}$ is likely to spur substantial research into the physical structure and properties of such composites. 


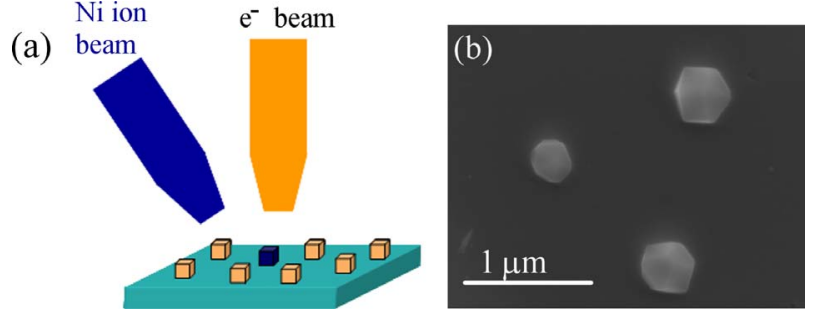

FIG. 1. (Color online) (a) Schematic of the SEM/FIB with nickel-ion source. The yellow (light gray) cubes represent diamond unimplanted diamond crystals while the blue (dark gray) one is the only crystal which was implanted with nickel. (b) SEM image of typical CVD diamond crystal after growth.

\section{EXPERIMENTAL DETAILS}

The diamond crystals were grown using a microwave CVD technique following a previously reported procedure. ${ }^{17}$ Briefly, the substrate (sapphire or silica cover slip) was seeded with nanodiamond powder (4-6 nm, Nanoamor Inc., Houston, Texas, USA) without ultrasonic treatment followed by a diamond growth using microwave plasma (900 W and 150 torr). After growth, the substrate was transferred to a dual SEM/FIB (Jeol JSM 5910) to perform the nickel implantation. Figure 1(a) shows a schematic illustration of the dual SEM/FIB used to implant the nickel into the diamond crystals. The surface was first imaged by the SEM to determine the diamond density and location. After choosing a specific crystal in a preferred location, a nickel FIB beam with a typical 1-5 pA current was used to perform the implantation into the same crystal. The nickel ions were isolated from the $\mathrm{Ni} / \mathrm{Er}$ source by a set of electromagnetic lenses and apertures located in the FIB column. The current was adjusted by moving the apertures in relation to the main ion beam, thus blocking part of the beam. The origin of the nickel ions is a Ni/Er liquid metal-ion source, ${ }^{18}$ and the selection of a specific ion type and energy is achieved by applying a set of apertures and an electromagnetic filter. The spot size of the beam can be reduced down to ten nanom- eters, allowing implantation into an individual CVD grown diamond crystal. Nickel ions were implanted with an energy of $30 \mathrm{keV}$. According to stopping range of ions in matter (SRIM) simulations, ${ }^{19}$ the stopping range of $30 \mathrm{keV}$ nickel ions is around $20 \mathrm{~nm}$ beneath the diamond surface. After implantation, the crystals were annealed at $1000{ }^{\circ} \mathrm{C}$ in $95 \% \mathrm{Ar}-5 \% \mathrm{H}_{2}$ ambient for $1 \mathrm{~h}$. Time correlation of photoluminescence (PL) intensity was performed with a HanburyBrown and Twiss (HBT) setup consisting of two avalanche photodiodes (APDs) in a single-photon counting regime placed on each side of a beam splitter. For this measurement we used a continuous-wave (cw) laser diode excitation source at a wavelength of $687 \mathrm{~nm} .{ }^{20}$ The collected light from the color centers then passed through a dichroic mirror (DM) and optical filters to remove any residual excitation light. A spectrometer (MicroHR, HORIBA Jobin Yvon) was used to record a photoluminescence spectrum from each individual center.

\section{RESULTS}

Figure 1(b) shows an SEM image of the grown diamond crystal. Using our seeding method, ${ }^{17}$ we are able to control the surface density of crystals on the substrate, thus allowing easy imaging by both the SEM/FIB and the detection using a confocal microscope. The diamonds were grown on a sapphire substrate to reduce the incorporation of silicon into the diamond crystal, which is commonly observed when silicon substrates are used. The diamond crystals were grown to the size of about 200-600 nm, which is small enough to eliminate the total internal reflection of the light, thereby enhancing emitted photon collection efficiency.

A scan over the post-implanted and annealed region using a confocal microscope revealed a bright fluorescence originating from the optical center created within the diamond. Figure 2(a) shows a PL spectrum taken at room temperature from an individual fluorescent diamond crystal, related to the formed nickel color center. A strong narrow-band luminescence (full width at half maximum $\sim 7 \mathrm{~nm}$ ) with no signifi-
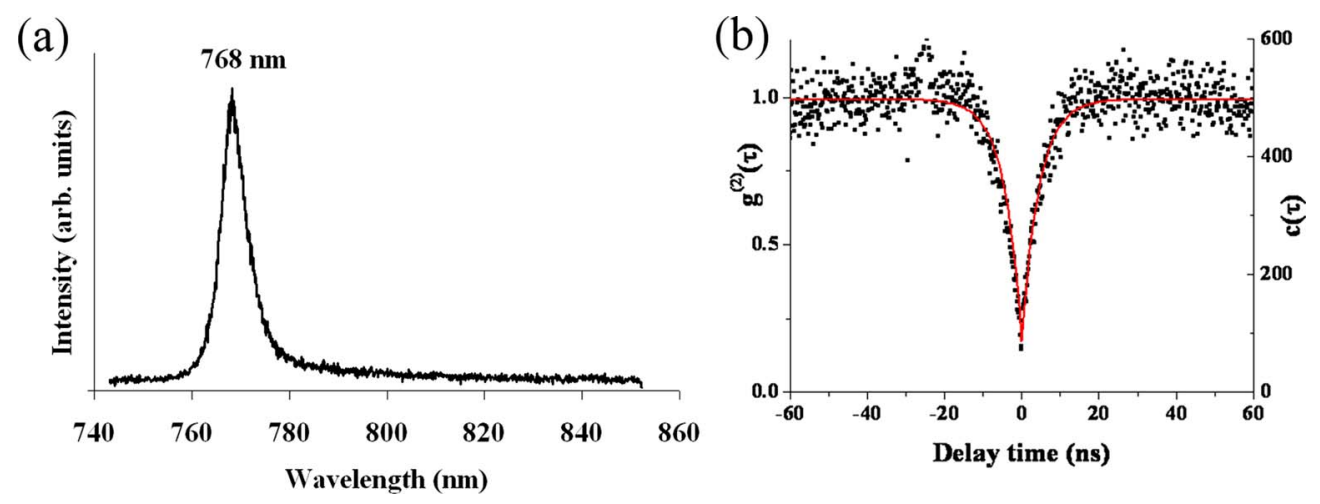

FIG. 2. (Color online) (a) PL spectrum from individual nickel-implanted diamond crystal under $687 \mathrm{~nm}$ excitation recorded at room temperature. (b) Corresponding normalized time autocorrelation function $g^{(2)}(\tau)$ of the PL signal recorded at room temperature. The excitation power is $100 \mu \mathrm{W}$ and the integration time is $300 \mathrm{~s}$ with a coincidence time bin of $154 \mathrm{ps}$. The dip at zero delay time with $g^{(2)}(0)=0.16$ indicates single-photon emission. The dots indicate the experimental data while the solid line is a fit of $g^{(2)}(\tau)$ taking into account background, instrumental response function due to finite time resolution of the APDs, and the correlation electronics. The slight bump at $\tau=-30 \mathrm{~ns}$ is due to remaining cross talk. 
cant phonon side band is observed at $768 \mathrm{~nm}$. More than $90 \%$ of the emission intensity is concentrated in the zero phonon line (ZPL). This center does not photoluminesce at the same wavelength as the previously reported NE8 or one of its variants. ${ }^{8,15,20}$ Almost all the implanted crystals revealed a similar emission peak around $770 \mathrm{~nm}$ while no PL was detected in the unimplanted regions, convincingly indicating that the origin of the signal is due to the FIB nickel implantation.

The photon statistics of PL light from these Ni-related color centers was studied by measuring the normalized second-order time autocorrelation function, $g^{(2)}(\tau)=\langle I(t) I(t$ $+\tau)\rangle /\langle I(t)\rangle^{2}$, using the HBT interferometer. ${ }^{21,22}$ Figure 2(b) shows the normalized second-order autocorrelation function recorded at room temperature from a color center in an individual diamond crystal located in the implanted area excited via cw laser, with the emission spectrum shown in Fig. 2(a). During all the measurements, the center was stable and no photobleaching or photoblinking behavior was observed. The dip of $g^{(2)}(\tau)$ at zero delay time indicates that the observed nickel center is indeed a single-photon emitter. Single-photon characteristics were found in crystals implanted with a dose of $5 \times 10^{10} \mathrm{Ni} / \mathrm{cm}^{2}$. This dose equals to $10-100 \mathrm{Ni}$ ions per crystal, depending on the exact orientation and the size of the crystal. Higher implantation doses (of $\sim 10^{11} \mathrm{Ni} / \mathrm{cm}^{2}$ ) produced double emitters with a $g^{(2)}(0)$ $\sim 0.5$ and emitters exhibiting Poissonian emission, i.e., $g^{(2)}(0) \sim 1$.

We also recorded the $g^{(2)}(\tau)$ function with increased cw laser excitation power to measure the decay time of the emitter. By extrapolating a linear fit to a zero excitation power, the lifetime of the center is calculated to be as short as 2 ns. This value is six times faster than the typical lifetime of an NV center in a CVD crystal and thus makes this nickelrelated center attractive for quantum photonic applications with a theoretical emission rate as fast as $0.5 \mathrm{GHz}$ operating at room temperature. This value is close to currently developed high-speed QKD systems with clock rates of $2 \mathrm{GHz}{ }^{23}$ The fluorescence intensity was measured as a function of laser power to evaluate the emission rate at saturation (Fig. 3 ). A high emission rate of $140 \mathrm{kcounts} / \mathrm{s}$ was measured with only one APD on the HBT setup. From the fit curve (Fig. 3), the total count rate at saturation power is therefore estimated to be greater than $200 \mathrm{kcounts} / \mathrm{s}$. Optimization of the diamond growth substrate, for example, using a highly reflective mirror ${ }^{2}$ or coupling to waveguide structures, ${ }^{24,25}$ should then lead to the realization of a very efficient single-photon source.

The quantum efficiency of the center can be estimated using

$$
\eta_{Q}=\frac{R_{\mathrm{sat}} \tau}{\eta_{\mathrm{det}}},
$$

where $R_{\text {sat }}$ is the count rate at saturation, $\tau$ is the excited-state lifetime, and $\eta_{\text {det }}$ is the total optical-detection efficiency. From the theoretical fit to the experimental data in Fig. 3, $R_{\text {sat }}=200 \mathrm{kcounts} / \mathrm{s}$, the excited-state lifetime of the center was measured to be 2 ns while the detection efficiency was estimated by determining the efficiencies of each component

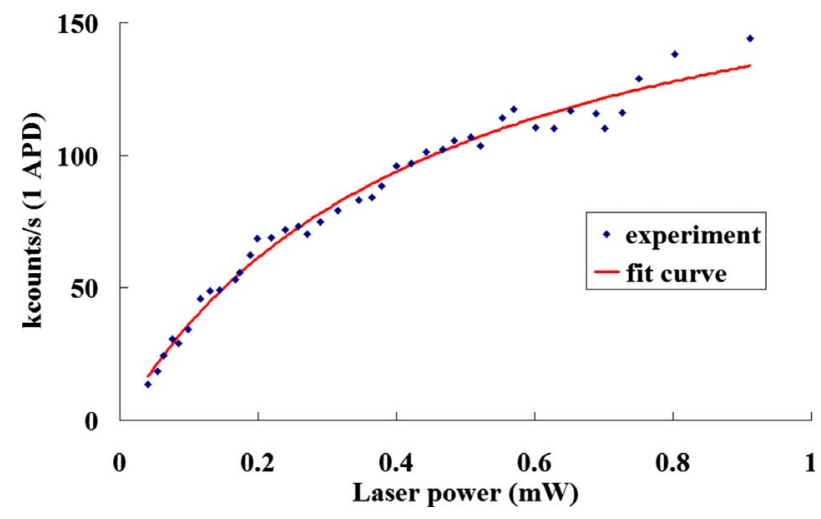

FIG. 3. (Color online) Counting-rate data corresponding to the background corrected PL intensity from the same single Ni-related color center shown in Fig. 2 as a function of excitation power. The background counting rate is $1.8 \mathrm{kcounts} / \mathrm{s}$ for an excitation power of $0.9 \mathrm{~mW}$.

in the collection system as detailed in Refs. 20 and 21, in which case $\eta_{\text {det }} \sim 0.04 \%$. This equates to an estimated quantum efficiency of $\sim 100 \%$, which suggests that the short lifetime of the center is associated with a strong radiative-decay component with a low probability of nonradiative decay.

Finally, we discuss the atomistic structure of the centers. It is clear from our experimental data that the observed center is not the previously reported nickel nitrogen, NE8 center. The luminescence was observed only from implanted crystals and, therefore, it is likely that the centers are nickel related. Previous work reported various emission lines characteristic of nickel impurities in diamond; ${ }^{26}$ however, an emission around $770 \mathrm{~nm}$ was not observed or assigned to any particular nickel-related center so far. Besides nitrogen, the most common and favorably incorporated impurity is silicon, which forms a common $\mathrm{Si}-\mathrm{V}$ center within the diamond lattice. Therefore, we assume that the center is related to both nickel and silicon complexes. To check our assumptions, we performed a control experiment by implanting both silicon and nickel into pure synthetic, type IIa, $3 \times 3 \times 0.5 \mathrm{~mm}^{3}$ diamond produced by element 6 (e6 Inc., U.K.). Nickel ions were implanted with an energy of $37.5 \mathrm{keV}$ while silicon ions were implanted using $25 \mathrm{keV}$. According to SRIM simulations, ${ }^{19}$ the stopping range of both nickel and silicon ions at these energies are around $18 \mathrm{~nm}$ beneath the diamond surface. The implantation was followed by the same annealing procedure as used on our CVD grown diamond crystals. A similar PL line centered at $766 \mathrm{~nm}$ was observed [Fig. 4(c)] while a nickel only implantation into the same crystal did not result in the formation of this specific emission line [Fig. 4(b)]. Note that the doublet at around 883 and $885 \mathrm{~nm}$ is associated with an interstitial nickel defect in diamond, ${ }^{27}$ and appears in both performed nickel implantations.

A similar vibronic feature around $768 \mathrm{~nm}$ was previously assigned to a local defect mode ${ }^{28}$ or to a local phonon mode of the Si-V center. ${ }^{29}$ It is important to note that in both cases the diamond origin for the CVD growth may have contained nickel impurities. In Ref. 28 it was a type Ib Sumitomo diamond while in Ref. 29 it was a commercial diamond powder. Our antibunching data clearly demonstrates that the center is 


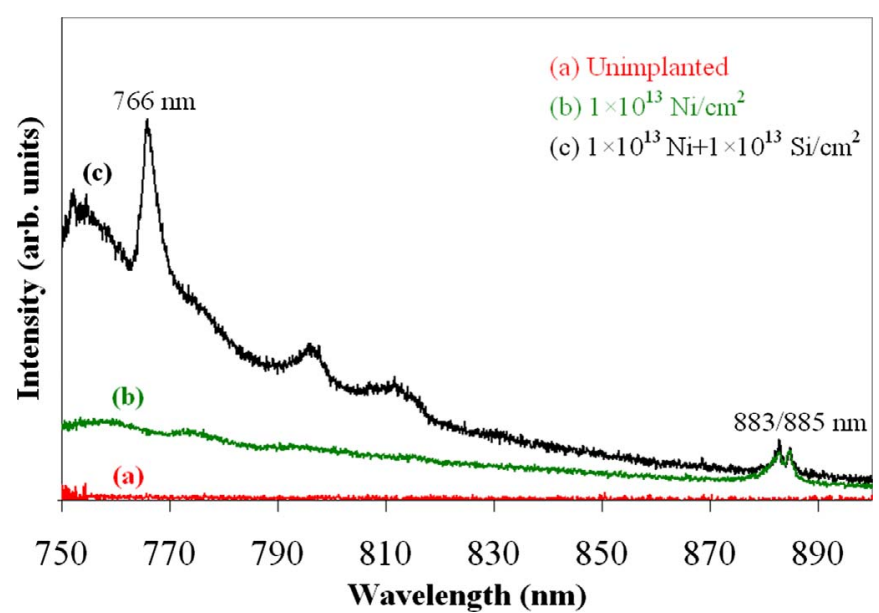

FIG. 4. (Color online) PL spectra from (a) an unimplanted region, (b) Ni only implanted region, and (c) a coimplanted $\mathrm{Ni} / \mathrm{Si}$ region of a type IIa e6 CVD diamond. The measurement is taken at $77 \mathrm{~K}$ under $514 \mathrm{~nm}$ excitation.

not a $\mathrm{Si}-\mathrm{V}$ center due to higher lifetime and high count rate, ${ }^{9}$ and our implantation technique shows that nickel appears to play a crucial role in enabling the emission. Therefore, we believe that the PL line at around $770 \mathrm{~nm}$ is due to a center containing both nickel and silicon atoms. Accordingly we propose the name UM1 for this center. Note that nickel and silicon each create a large distortion in the diamond crystal lattice. Hence, it is possible that upon annealing a vacancy is combined with those two atoms, and the actual structure consists of Si and Ni impurities associated to a vacancy. Given that the CVD diamonds do not show NV emission, it is unlikely that the complex also contains N. The source of the silicon inside the grown CVD diamond crystals may possibly originate from the quartz made CVD chamber. An incorporation of a few ppm of silicon into the diamond crystals during the growth is hence likely. The concentration might be too low to be observed as $\mathrm{Si}-\mathrm{V}$ because that center is not an efficient emitter but when it complexes with an implanted nickel ion, it may lead to the formation of the observed very- bright centers, even for very low silicon concentrations. Investigations of the atomistic structure and theoretical simulations will be the subject of future work.

\section{CONCLUSIONS}

In summary, we have engineered a highly efficient singlephoton emitter based on nickel complex in individual CVD diamond crystal, which we have designated as UM1. It possesses a short lifetime of only 2 ns and near-unity quantum efficiency, which resulted in a total high count rate (from two APDs) of more than $200 \mathrm{kHz}$ at room temperature. Centers were formed via focused ion beam nickel implantation into selected diamond crystals which gives rise to high-potential spatial accuracy. The combination of room-temperature operation, high brightness, and step and repeat fabrication compatibility makes UM1 an attractive solid-state candidate to be used in integrated quantum optics. Coimplantation studies using nickel and silicon strongly suggest that UM1 is a hybrid nickel-silicon complex. Moreover, the coimplantation of ions opens new possibilities and new defect centers to be explored as building blocks for quantum optical engineering networks.

\section{ACKNOWLEDGMENTS}

This work was supported by the Australian Research Council, The International Science Linkages Program of the Australian Department of Innovation, Industry, Science and Research, the European Union under the EQUIND (Project No. IST-034368) and NEDQIT (ERANET NanoSci) projects, and by the French Agence Nationale de la Recherche PROSPIQ project (Project No. ANR-06-NANO041). A.D.G. acknowledges financial support from the Australian Research Council (Project No. DP0880466). I.A. acknowledges ARNAM and ARC for their financial support and C.Z. acknowledges support from the Foundation EADS. The authors are thankful to Daniel Twitchen for helpful discussions, and to Wolfgang Pilz and Lothar Bischoff for the help with the FIB.

\footnotetext{
*Corresponding author; i.aharonovich@pgrad.unimelb.edu.au

${ }^{1}$ A. Gruber, A. Drabenstedt, C. Tietz, L. Fleury, J. Wrachtrup, and C. von Borczyskowski, Science 276, 2012 (1997).

${ }^{2}$ A. Beveratos, R. Brouri, T. Gacoin, A. Villing, J.-P. Poizat, and P. Grangier, Phys. Rev. Lett. 89, 187901 (2002).

${ }^{3}$ V. Jacques, E. Wu, F. Grosshans, F. Treussart, P. Grangier, A. Aspect, and J.-F. Roch, Science 315, 966 (2007).

${ }^{4}$ http://qcvictoria.com.

${ }^{5}$ A. D. Greentree, B. A. Fairchild, F. M. Hossain, and S. Prawer, Mater. Today 11, 22 (2008).

${ }^{6}$ C. L. Degen, Appl. Phys. Lett. 92, 243111 (2008); J. R. Maze, P. L. Stanwix, J. S. Hodges, S. Hong, J. M. Taylor, P. Cappellaro, L. Jiang, M. V. Gurudev Dutt, E. Togan, A. S. Zibrov, A. Yacoby, R. L. Walsworth, and M. D. Lukin, Nature (London) 455 , 644 (2008); G. Balasubramanian, I. Y. Chan, R. Kolesov, M.
}

Al-Hmoud, J. Tisler, C. Shin, C. Kim, A. Wojcik, P. R. Hemmer A. Krueger, T. Hanke, A. Leitenstorfer, R. Bratschitsch, F. Jelezko, and J. Wrachtrup, ibid. 455, 648 (2008).

${ }^{7}$ S. J. Devitt, A. D. Greentree, R. Ionicioiu, J. L. O’Brien, W. J. Munro, and L. C. L. Hollenberg, Phys. Rev. A 76, 052312 (2007).

${ }^{8}$ T. Gaebel, I. Popa, A. Gruber, M. Domhan, F. Jelezko, and J. Wrachtrup, New J. Phys. 6, 98 (2004).

${ }^{9}$ C. Wang, C. Kurtsiefer, H. Weinfurter, and B. Burchard, J. Phys. B 39, 37 (2006).

${ }^{10}$ F. C. Waldermann, P. Olivero, J. Nunn, K. Surmacz, Z. Y. Wang, D. Jaksch, R. A. Taylor, I. A. Walmsley, M. Draganski, P. Reichart, A. D. Greentree, D. N. Jamieson, and S. Prawer, Diamond Relat. Mater. 16, 1887 (2007).

${ }^{11}$ J. R. Rabeau, P. Reichart, G. Tamanyan, D. N. Jamieson, S. 
Prawer, F. Jelezko, T. Gaebel, I. Popa, M. Domhan, and J. Wrachtrup, Appl. Phys. Lett. 88, 023113 (2006).

${ }^{12}$ J. Martin, R. Wannemacher, J. Teichert, L. Bischoff, and B. Kohler, Appl. Phys. Lett. 75, 3096 (1999).

${ }^{13}$ C.-H. Su, A. D. Greentree, W. J. Munro, K. Nemoto, and L. C. L. Hollenberg, Phys. Rev. A 78, 062336 (2008).

${ }^{14}$ D. N. Jamieson, C. Yang, T. Hopf, S. M. Hearne, C. I. Pakes, S. Prawer, M. Mitic, E. Gauja, S. E. Andresen, F. E. Hudson, A. S. Dzurak, and R. G. Clark, Appl. Phys. Lett. 86, 202101 (2005).

${ }^{15}$ J. R. Rabeau, Y. Chin, S. Prawer, F. Jelezko, T. Gaebel, and J. Wrachtrup, Appl. Phys. Lett. 86, 131926 (2005).

${ }^{16}$ I. Aharonovich, C. Zhou, A. Stacey, F. Treussart, J.-F. Roch, and S. Prawer, Appl. Phys. Lett. 93, 243112 (2008).

${ }^{17}$ A. Stacey, I. Aharonovich, S. Prawer, and J. E. Butler, Diamond Relat. Mater. 18, 51 (2009).

${ }^{18}$ L. Bischoff, Ultramicroscopy 103, 59 (2005).

${ }^{19}$ The stopping and range of ions in matter, www.srim.org.

${ }^{20}$ E. Wu, V. Jacques, H. Zeng, P. Grangier, F. Treussart, and J.-F. Roch, Opt. Express 14, 1297 (2006).
${ }^{21}$ The low detection efficiency is limited by the dichroic mirror which is not ideal for the emission around $770 \mathrm{~nm}$.

${ }^{22}$ R. Brouri, A. Beveratos, J.-Ph. Poizat, and P. Grangier, Opt. Lett. 25, 1294 (2000).

${ }^{23}$ K. J. Gordon, V. Fernandez, G. S. Buller, I. Rech, S. D. Cova, and P. D. Townsend, Opt. Express 13, 3015 (2005).

${ }^{24}$ J. R. Rabeau, S. T. Huntington, A. D. Greentree, and S. Prawer, Appl. Phys. Lett. 86, 134104 (2005).

${ }^{25}$ K.-M. C. Fu, C. Santori, P. E. Barclay, I. Aharonovich, S. Prawer, N. Meyer, A. M. Holm, and R. G. Beausoleil, Appl. Phys. Lett. 93, 234107 (2008).

${ }^{26}$ A. Yelisseyev, S. Lawson, I. Sildos, A. Osvet, V. Nadolinny, B. Feigelson, J. M. Baker, M. Newton, and O. Yuryeva, Diamond Relat. Mater. 12, 2147 (2003).

${ }^{27}$ K. Iakoubovskii and G. Davies, Phys. Rev. B 70, 245206 (2004), and references therein.

${ }^{28}$ H. Sternschulte, K. Thonke, R. Sauer, P. C. Munzinger, and P. Michler, Phys. Rev. B 50, 14554 (1994).

${ }^{29}$ T. Feng and B. D. Schwartz, J. Appl. Phys. 73, 1415 (1993). 